\title{
Case Report \\ Neuroleptic Malignant Syndrome-an uncommon complication of a common drug- a case report
}

\author{
Vaishnavi Danasekaran, S. Lokesh, Prasanna Venkatesh, Aftab
}

Department of General Medicine, Mahatma Gandhi Medical College and Research Institute, Sri Balaji Vidhyapeeth (deemed to be) University, Pillaiyarkuppam, Puducherry, India

\author{
(Received: August $2020 \quad$ Revised: December $2020 \quad$ Accepted: December 2020)
}

Corresponding author: S. Lokesh. Email: lokeshsdr@gmail.com

\begin{abstract}
Neuroleptic Malignant Syndrome (NMS) is a life-threatening idiosyncratic reaction to neuroleptics and rarely to antidopaminergics. Although the incidence in India is $0.14 \%$, it is potentially a fatal complication. NMS occurs due to a blockade in the dopamine receptor and nigrostriatal pathway. The following report is about our patient who was diagnosed with NMS. This patient was diagnosed of NMS due to a progressive rise in CPK NAC and liver enzymes, metabolic acidosis and pyrexia of unknown origin not responding to treatment. No foci for the cause of pyrexia were ascertained. However, a positive drug history of recent administration of Inj. Metaclopramide was the present treatment in the form of supportive care, by controlling rigidity, hyperthermia and preventing complications that can occur following the affliction of this condition. NMS is a potentially reversible fatal complication with a mortality rate of 30\%. Indiscriminate usage of neuroleptics and antidopaminergics can inadvertently lead to patients developing this condition. This case has been reported to create awareness amongst physicians about this complication.
\end{abstract}

Keywords: Neuroleptic malignant syndrome; acute undifferentiated febrile illness; treatment of NMS; fever with muscle rigidity

\section{INTRODUCTION}

$\mathrm{N}$ euroleptic Malignant Syndrome is a rarely reported but serious complication of neuroleptic medications. It was first described in 1967 as "akinetic hypertonic syndrome". Even as a well-known entity, this clinical syndrome is often missed because of its varied and subtle clinical manifestation. The prognosis of this clinical entity improved dramatically because of early identification and prompt management but still with the mortality of $10 \%$. In this case report we report an elderly female with Neuroleptic Malignant Syndrome.

\section{CASE REPORT}

A 54-year-old female presented to casualty with high grade fever and altered sensorium. She had recently been administered a few doses of Inj. Metoclopramide for nausea for a duration of 3 days, from a local physician, following which, she developed episodes of altered behaviour and irrelevant speech of 1-day duration. She resides with her husband, who is bedridden. Her drug history was not reliable as well.

On general examination, she was found to be dehydrated, confused, febrile and tachypnoeic. There was no eschar / rash / bite marks. No signs suggestive of meningeal irritation were present . Her vital parameters included a regular pulse rate of $130 /$ minute, $\mathrm{BP}$ of $130 / 90 \mathrm{~mm} \mathrm{Hg}, \mathrm{RR}$ of $22 /$ minute and a temp. of $102^{\circ} \mathrm{F}$. Neurological examination displayed normal tone and reflexes in all four limbs with down-going plantars. Pupillary reaction to light was $2 \mathrm{~mm}$. All other systemic examination was found to be normal.
Investigations showed metabolic acidosis with hypoxia, with a normal hemogram and deranged LFT. Fever panel was negative. CXR showed clear lung fields with no abnormalities. Lumbar puncture was inconclusive. Patient was then started on a course of empirical antibiotics, antivirals and antifungals consisting of Inj. Ceftriaxone $2 \mathrm{~g}$ iv bd, Inj Acyclovir $800 \mathrm{mg}$ tds, T. Fluconazole $400 \mathrm{mg}$ od.

On the following day, the patient developed generalized rigidity with persistent high-grade fever $\left(104-105^{\circ} \mathrm{F}\right)$, that did not touch baseline, along with diaphoresis and tremors. She also displayed features of autonomic instability, evidenced by persistent tachycardia and fluctuations in blood pressure.

Creatine phosphokinase N-acetyl cysteine (CPKNAC) was found to be elevated at 3290U/L. Echocardiogram showed no RWMA, a normal LV systolic function with an $\mathrm{EF}$ of $62 \%$ and no vegetations. CT Brain (plain) showed no evidence of infarcts or intracerebral haemorrhage/SOL. MRI Brain with contrast showed age related cerebral atrophic changes with no evidence of any leptomeningeal enhancement.

Serial CPK NAC levels further increased to 7710U/L along with altered liver enzyme parameters (AST and ALT of $353 \mathrm{U} / \mathrm{L}$ and $174 \mathrm{U} / \mathrm{L}$ respectively) and renal dysfunction, in the form of a raised creatinine, of more than $50 \%$ of the baseline value. Urine myoglobin was $731 \mathrm{microgram} / \mathrm{l}$.

Neuroleptic malignant syndrome (NMS) was suspected, according to DSM-IV criteria and the patient was intubated in view of severe metabolic 
acidosis, autonomic instability and hypoxia. She was started on a stat dose of Dantrolene Sodium $200 \mathrm{mg}$ followed by $80 \mathrm{mg}$ QID. Adequate hydration was maintained by administration of intravenous fluids and application of external cooling blankets.

Once the treatment was focused on NMS, CPK NAC values dropped significantly to $2706 \mathrm{U} / \mathrm{L}$, liver enzymes, metabolic parameters and renal function improved, and her rigidity reduced. All parameters returned to baseline in two days after the commencement of treatment, with residual prevalence of mild autonomic disturbances.

\section{DISCUSSION}

Neuroleptic malignant syndrome is a rare but fatal complication, that can occur following the administration of neuroleptics. The pathophysiology behind this condition being dopamine D2 receptor antagonism, as serotonin stimulation is inhibited leading to extreme hyperthermia. A blockade in the central D2 receptor in the hypothalamus, nigrostriatal pathways and spinal cord leads to increased muscle rigidity and tremor via the extrapyramidal pathway. A blockade in the nigrostriatal pathway results in rigidity and inhibition of the mesolimbic/mesocortical pathways lead to an altered mental status. Furthermore, peripherally acting antipsychotics cause an increased release of calcium from the sarcoplasmic reticulum, leading to increased muscular contractility.

Psychomotor agitation occurs as a preceding symptom prior to the onset of clinical signs of NMS (1). An associated presence of dehydration in patients medicated with neuroleptics, acts as a significant risk factor for developing NMS, as it leads to an impairment in temperature regulation. Adequate hydration of such susceptible patients acts as a useful adjunct to treatment (2). NMS can occur within a wide time period. A large proportion of cases develop within the first week, following the commencement of antipsychotic treatment, with more than $90 \%$ of cases occurring within the first four weeks (3). Important complications to keep in mind while treating such patients include renal failure secondary to myoglobinuria, cardiac arrhythmias, hypoxia, pulmonary embolism and prolonged hyperpyrexia. Metoclopramide, promethazine and prochlorperazine are some of the drugs that are known to cause this devastating condition $(4,5)$. To compensate for the dopamine blockade, an excess of noradrenaline is released to balance its effect, which in turn leads to a hypermetabolic condition, that is classically seen in such patients. Genetic predisposition plays a crucial role and seeking a family history is justified in such scenarios (6). Leukocytosis, elevated CPK NAC, metabolic acidosis and dyselectrolytemia are the most commonly encountered laboratory findings (5).

NMS is a diagnosis of exclusion. It is crucial to rule out other medical and neurological conditions that have similar clinical presentations especially in the older population (7). Heat stroke and serotonin syndrome are a few entities that are often confused with NMS. Patients with heat stroke present with hyperthermia and dry skin with loss of muscle tone. However, NMS causes hyperthermia associated with generalized muscle rigidity and diaphoresis (8). Adequate hydration, correction of electrolyte abnormalities and metabolic acidosis, along with the appropriate usage of adjuncts to reduce hyperthermia, whilst monitoring vital organ functions forms the basis of treatment ${ }^{5}$. Usage of benzodiazepines such as lorazepam have also been found to be useful to prevent the onset of extrapyramidal symptoms (9). Administration of Dantrolene sodium at $2-3 \mathrm{mg} / \mathrm{kg}$ body weight becomes necessary when core body temperature exceeds 40C. Dantrolene sodium acts a skeletal muscle relaxant by blocking receptors that are specific for the release of calcium in skeletal muscles, bringing about a reduction in the excitationcontraction coupling mechanism (10). Once the patient begins to respond to treatment, the dose can be reduced to $1 \mathrm{mg} / \mathrm{kg}$ body weight QID for an additional 10 days and gradually tapered off. Usage of this drug however necessitates careful monitoring of GCS as well as serial LFTs as they are known to cause a depression of the central nervous system derangement of liver parameters (11).

\section{CONCLUSION}

Most often the diagnosis of Neuroleptic Malignant Syndrome is mainly clinical supported by lab investigations. The drug history which is often forgotten in the clinical practice stands the main clinching point in the diagnosis. Most of the drugs which are associated with Neuroleptic Malignant Syndrome are mainly dopamine antagonist which are commonly prescribed in day-to-day clinical practice. Understanding the pathophysiology and clinical manifestations makes it easy to differentiate from the other close differentials. Prevention is better than cure, like wise a slow and gradual dose titration of the precipitating drug most often prevents the incidence of Neuroleptic Malignant Syndrome even in high-risk individuals.

\section{CONFLICT OF INTEREST}

Authors declare no conflict of interest.

\section{REFERENCES}

1. Keck, P. E., Pope, H. G., Cohen, B. M., McElroy, S. L., Nierenberg, A. A. Risk factors for neuroleptic malignant syndrome. A case-control study. Arch Gen Psychiatry. 1989 Oct; 46(10): 914-918.

2. Bond, W. S. Detection and management of the neuroleptic malignant syndrome. Clin Pharm. 1984 Jun; 3(3): 302-307.

3. Caroff, S. N., Mann, S. C. Neuroleptic malignant syndrome. Med Clin North Am. 1993 Jan; 77(1): 185-202.

4. Velamoor, R., Neuroleptic malignant syndrome: A neuropsychiatric emergency: Recognition, prevention, and management. Asian Journal of Psychiatry. 2017 Oct; 29: 106-109. 
5. Velamoor, V. R. Neuroleptic malignant syndrome. Recognition, prevention and management. Drug Saf. 1998 Jul; 19(1): 73-82.

6. Kawanishi, C. Genetic predisposition to neuroleptic malignant syndrome: implications for antipsychotic therapy. Am J Pharmacogenomics. 2003; 3(2):89-95.

7. Sewell, D. D., Jeste, D. V. Distinguishing neuroleptic malignant syndrome (NMS) from NMS-like acute medical illnesses: a study of 34 cases. J Neuropsychiatry Clin Neurosci. 1992; 4(3): 265-269.

8. Caroff, S. N., Campbell, E. C., Sullivan, K. A. Neuroleptic malignant syndrome in elderly patients. Expert Rev Neurother. 2007 Apr; 7(4): 423-431.

9. Woodbury, M. M., Woodbury, M. A. Neuroleptic-induced catatonia as a stage in the progression toward neuroleptic malignant syndrome. J Am Acad Child Adolesc Psychiatry. 1992 Nov; 31(6): 1161-1164.

10. Krause, T., Gerbershagen, M. U., Fiege, M., Weisshorn, R., Wappler, F., Dantrolene - a review of its pharmacology, therapeutic use and new developments. Anaesthesia. 2004 Apr; 59(4): 364-373.

11. Sakkas, P., Davis, J. M., Janicak, P. G., Wang, Z. Y. Drug treatment of the neuroleptic malignant syndrome. Psychopharmacol Bull. 1991; 27(3): 381-384. 